\title{
Numerical Simulation Analysis of an Oversteer In-Wheel Small Electric Vehicle Integrated with Four-Wheel Drive and Independent Steering
}

\author{
Muhammad Izhar Ishak, ${ }^{1}$ Hirohiko Ogino, ${ }^{2}$ and Yoshio Yamamoto ${ }^{3}$ \\ ${ }^{1}$ Course of Science and Technology, Tokai University, 4-1-1 Kitakaname, Hiratsuka-shi, Kanagawa 259-1292, Japan \\ ${ }^{2}$ Department of Prime Mover Engineering, Tokai University, 4-1-1 Kitakaname, Hiratsuka-shi, Kanagawa 259-1292, Japan \\ ${ }^{3}$ Department of Precision Engineering, Tokai University, 4-1-1 Kitakaname, Hiratsuka-shi, Kanagawa 259-1292, Japan \\ Correspondence should be addressed to Muhammad Izhar Ishak; m.izhar.ishak@gmail.com
}

Received 22 February 2016; Revised 1 May 2016; Accepted 11 May 2016

Academic Editor: Shankar Subramanian

Copyright ( 2016 Muhammad Izhar Ishak et al. This is an open access article distributed under the Creative Commons Attribution License, which permits unrestricted use, distribution, and reproduction in any medium, provided the original work is properly cited.

\begin{abstract}
Similar to conventional vehicle, most in-wheel small EVs that exist today are designed with understeer (US) characteristic. They are safer on the road but possess poor cornering performance. With recent in-wheel motor and steer-by wire technology, high cornering performance vehicle does not limit to sport or racing cars. We believe that oversteer (OS) design approach for in-wheel small EV can increase the steering performance of the vehicle. However, one disadvantage is that OS vehicle has a stability limit velocity. In this paper, we proposed a Four-Wheel Drive and Independent Steering (4WDIS) for in-wheel small EV with OS characteristic. The aim of implementing 4WDIS is to develop a high steer controllability and stability of the EV at any velocity. This paper analyses the performance of OS in-wheel small EV with 4WDIS by using numerical simulation. Two cornering conditions were simulated which are (1) steady-state cornering at below critical velocity and (2) steady-state cornering over critical velocity. The objective of the simulation is to understand the behavior of OS in-wheel small EV and the advantages of implementing the 4WDIS. The results show that an in-wheel small EV can achieve high cornering performance at low speed while maintaining stability at high speed.
\end{abstract}

\section{Introduction}

In recent years, concerns over environmental issues by petroleum-based transportations along with the issue of fossil fuel depletion around the world have led to renewed interest over electric vehicle (EV). Small EVs have become prominent as a means of transportation, especially in urban areas where there are limited spaces $[1,2]$. The driving method of such an electric vehicle can be divided into two types. The most basic type is the central motor system, where a conventional combustion engine is replaced with an electric motor. The other type is the in-wheel motor system, where a driving motor is located near the hub of each wheel. We believe that the inwheel motor system has tremendous potential in the future of EVs for its compact size and high-energy efficiency. In past research, results show that the in-wheel motor system offers more freedom of movement for each wheel and can be governed separately by control systems such as the antilock brake system (ABS) and the traction control system (TCS) [3-5].

A few car manufacturers have marketed compact, cheap, and reliable in-wheel small electric vehicle that is aimed for daily usage. Similar to conventional vehicle, most in-wheel small EVs that exist today are designed with understeer (US) characteristic [6]. Even though they are safer on the road, they have poor cornering performance. With in-wheel motor and steer-by-wire technology nowadays, high cornering performance vehicle does not limit only sport or racing cars. We believe that oversteer (OS) design approach for in-wheel small EV can increase the steering response and lower the steering input with shorter cornering radius. However, one disadvantage of OS characteristic is that it has a stability limit velocity [7]. In this paper, we proposed a Four-Wheel Drive 


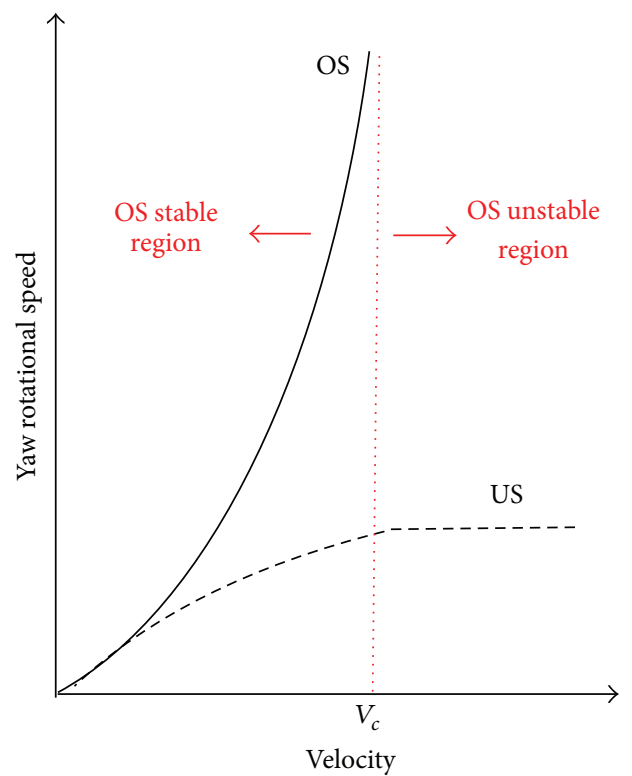

FIGURE 1: Steady-state yaw rotational speed to velocity between OS and US characteristic.

and Independent Steering (4WDIS) for in-wheel small EV that is designed with OS characteristic. The aim of implementing 4WDIS is to develop a high steer controllability and stability of an OS in-wheel small EV at any velocity.

This paper analyses the performance of 4WDIS on an OS in-wheel small EV by using numerical simulation. Two cornering conditions were simulated which are (1) steadystate cornering at below critical velocity and (2) steady-state cornering over critical velocity. The objective of the simulation is to understand the behavior of an OS in-wheel small EV and the advantages of implementing the 4WDIS. The 4WDIS is governed by an Intelligence Steering Control System (ISCS) that evaluates the dynamics of the $\mathrm{EV}$, the surrounding condition (i.e., narrow space), the road surface (i.e., dry, wet and icy road), and vehicle stability (i.e., crosswind and soft collision) in response to the drivers driving input. The ISCS will control the four-wheel driving and braking with each in-wheel motor and steer angle by the steering actuators to ensure safe maneuverability. The results show that, by implementing 4WDIS, an OS in-wheel small EV can achieve high cornering performance and maintain stability at any speed.

\section{Main Symbols}

Nomenclature shows the constant parameters and variables with the meaning, value, and unit used in this paper, respectively.

\section{Limitations of OS and US Characteristics}

A basic understanding of OS and US characteristic is necessary before any control method can be implemented. Figure 1 shows the relationship of the steady-state yaw rotational

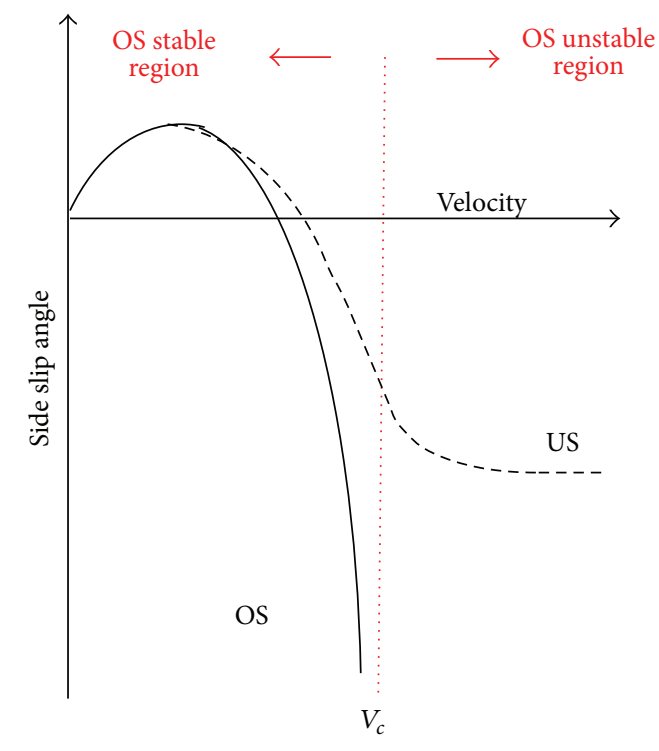

FIGURE 2: Steady-state side-slip angle to velocity between OS and US characteristic.

speed to velocity, and Figure 2 shows the relationship of steady-state side-slip angle to velocity between OS and US characteristic during steady-state cornering at constant steer angle. Based on the figure, the OS characteristic is separated into stable region and unstable region depending on the velocity of the vehicle. When the vehicle velocity $V$ is below the stability limit velocity $V_{c}$, OS vehicle can achieve steady-state cornering with a higher yaw rotational speed in comparison to US characteristic. A higher yaw rotational speed should also mean that the vehicle can achieve a shorter turning radius. The side-slip angle becomes negative as the absolute magnitude increases with velocity. This means that when the velocity increases, the vehicle will point to the inner side of the circular path of the cornering.

However, in the case of the velocity $V \geq V_{c}$, the yaw rotational speed and the side-slip angle will produce an infinite value with regard to a constant steer angle. It does not mean that the vehicle cannot be driven above this velocity but it is important to note that the vehicle motion is unstable. For this particular reason, most vehicles are manufactured with US characteristic that has no stability limit velocity as a compensation to low steering response. Nonetheless, US vehicle also has a tendency to cause accidents at high speed due to its restrictive steering.

\section{Four-Wheel Drive and Independent Steering (4WDIS) Approach}

4.1. Steering Characteristics. Two-wheel steering (2WS) or front wheel steering system (FWS) is generally known in all moving vehicles. This system works by rotating the front wheel in an angle that produces yaw motion, which then push the rear wheels with no steer angle sideways. A 2 WS vehicle with oversteer characteristic will have a shorter turning radius but it is difficult to stabilize with front wheel steering alone. 


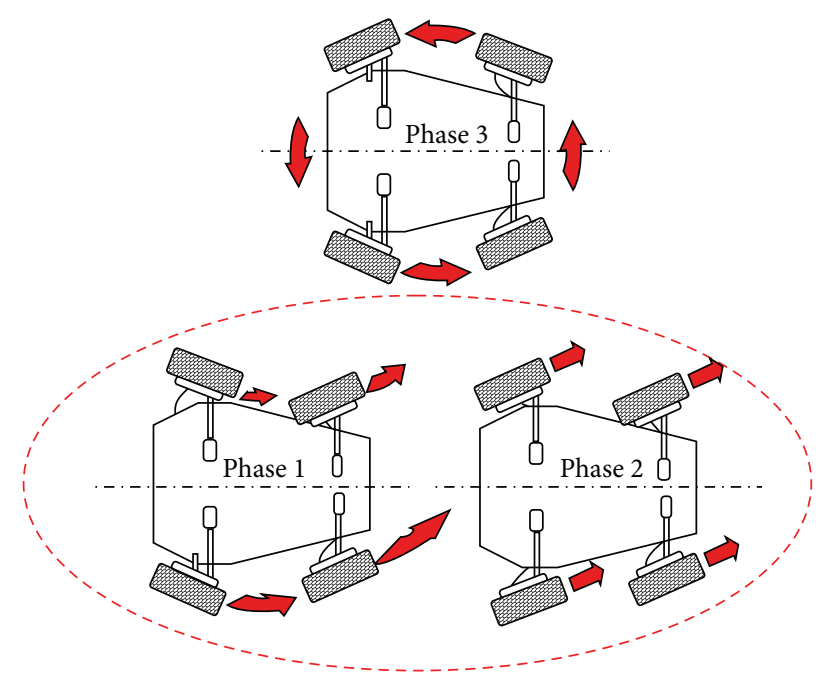

FIGURE 3: 4WDIS steering characteristics.

Four-wheel steering (4WS) has been introduced and marketed by Honda since 1987 in their Prelude model and then was joined by other car manufacturers to promote stability for conventional vehicle. The trend was short-lived due to the mechanical constrain of the system [8]. Nonetheless, with inwheel motor system and steer-by-wire technology, a small electric vehicle can have the freedom of design and layout to implement Four-Wheel Drive and Independent Steering (4WDIS). In this research, the main purpose of implementing 4WDIS is to have high cornering performance for OS inwheel small EV while maintaining stability at any speed [9]. Considering that each wheel's driving torque can be governed separately and the direction of the wheels can be manipulated with certain control, we had concluded that the steering modes for 4WDIS as in Figure 3 and the descriptions are as follows:

(a) Opposite Steering (Phase 1). The rear wheels will turn on the opposite direction as the front wheels. This will increase the vehicle yaw rotational speed, which tighten the turning radius.

(b) Parallel Steering (Phase 2). The rear wheels will rotate in the same direction parallel to the front. This steering does not generate yaw rotational speed but instead reduces the yaw rotational speed.

(c) "Zero-Radius" Steering (Phase 3). The front wheels have to be "toe-in" position and the rear wheels in "toe-out" position. The driving direction of the left and right is in opposite. This allows the vehicle to rotate at its yaw rotational center.

4.2. Intelligence Steering Control System (ISCS). An Intelligence Steering Control System (ISCS) is used to govern the 4WDIS. The ISCS evaluates the dynamics of the EV, the surrounding condition (i.e., narrow space), the road surface (i.e., dry, wet, and icy road), and vehicle stability (i.e., crosswind and soft collision) with regard to the drivers steering input. After the evaluation, the ISCS will choose the suitable

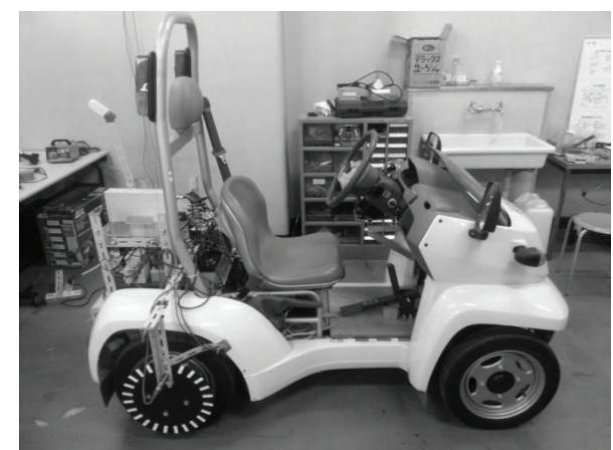

FIgURE 4: Analysis vehicle model of Toyota COMS.

steering characteristics to ensure stability and maneuverability.

In this paper, the evaluated parameter focuses on the dynamic of the in-wheel small EV and dry road surface. Thus, when studying the cornering performance of OS in-wheel small EV, only the alternation between phases 1 and 2 as shown in Figure 3 can be observed in the results.

\section{Analysis Vehicle Model}

Toyota COMS AK10E-PC shown in Figure 4, which is an inwheel small electric vehicle manufactured by Toyota Auto Body Co., was used as an analysis vehicle model in the simulation. The vehicle uses a $2 \mathrm{WD}$ in-wheel motor and has a front wheel steering. However, in the simulation, we added two in-wheel motors to the front wheels and two-steering actuator at the rear wheels. Thus, the mass of the vehicle model is increased from the actual vehicle. Furthermore, the weight distribution of the vehicle model was modified so that the weight shifted to the rear to represents a highly OS inwheel small EV while the dimensions of the vehicle remain the same.

\section{Simulation Block Diagram}

Figure 5 shows the simulation block diagram for the vehicle model and the ISCS. The vehicle model, which is an OS in-wheel small EV equipped with 4 WDIS, is calculated by a nonlinear system. Much of past researches adopted easy way of modelling a control system based on a vehicle with linear characteristics. By this assumption, other parameters that could influence the dynamic motion of the vehicle are neglected and the modelled control system is deemed to be low precision [10-13]. In this paper, the purpose of adopting nonlinear system for calculating the vehicle model is that the system represents more precisely an actual vehicle. However, a feedback control cannot be executed directly to nonlinear system because of yaw rotational speed coupling with the velocity components as expressed in dynamic equation of motion in later section. As an option, the ISCS, which is a state observer unit that consist of a feedback controlled linear system, is used to control the vehicle model. Each model in the simulation block will be explained in detail in the following sections. 


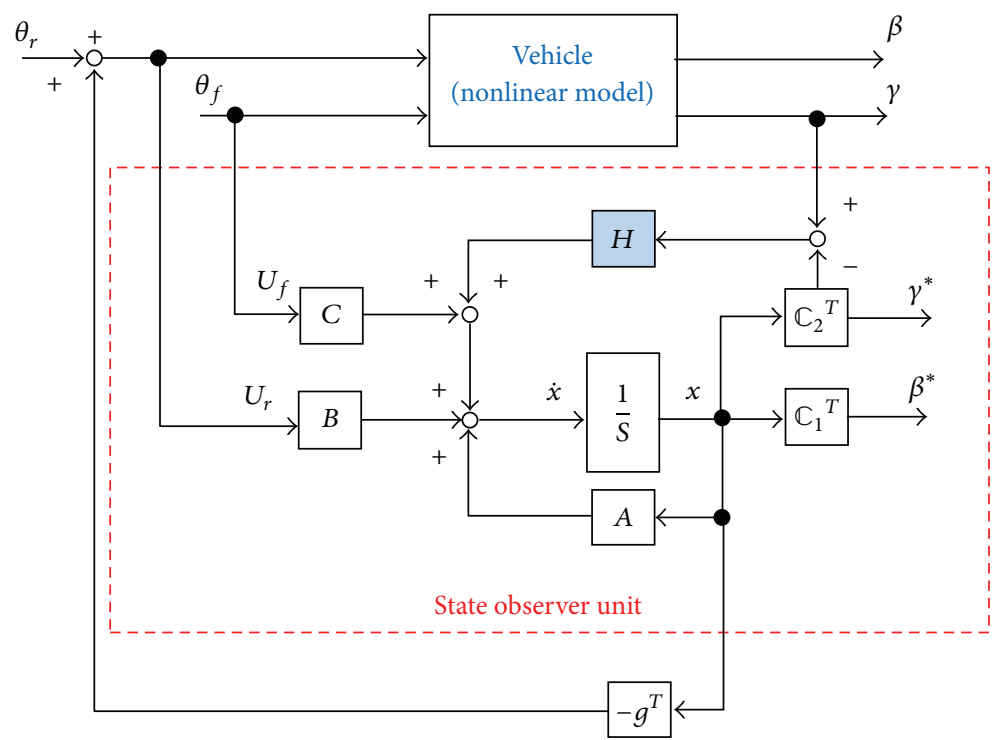

FIGURE 5: Simulation block diagram.

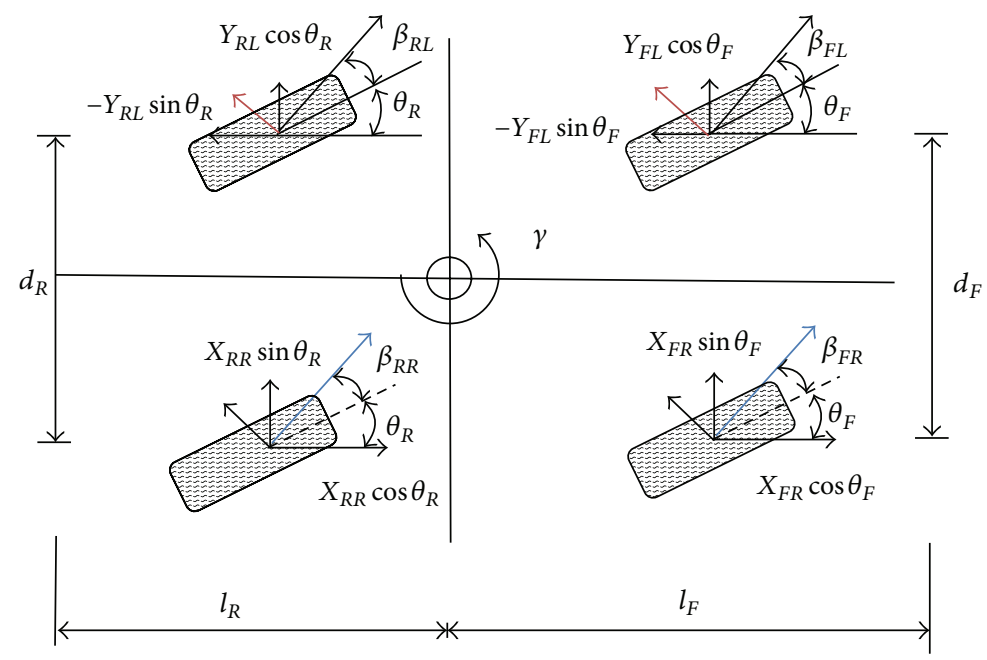

FIGURE 6: Force vector.

\section{Vehicle Model}

7.1. Nonlinear Dynamic Equations. The vehicle model has six degrees of freedom: three translational along the $x-, y$ , and $z$-axes and three rotational about the $x$-, $y$-, and $z$ axes. In this study, however, a planar motion is assumed so that a translation along the $z$-axis and rotations about the $x$ - and $y$-axes are disregarded. Figure 6 depicts the force vector used to construct the dynamic equations of motion for the vehicle and for the yaw rotational speed. Unlike linear dynamic equation, the vehicle side-slip angle at the center of gravity and wheels steer angle is not considered as small. Thus, we took the consideration that the longitudinal and lateral acceleration are influenced by the yaw rotational speed, which can be seen in (1).

The assumptions mentioned above necessitate calculating the dynamic equations for longitudinal and lateral velocities, including the coupling of the yaw rotation speed with the velocities:

$$
\begin{gathered}
m\left(\frac{d u}{d t}-v \gamma\right) \\
=\left(X_{F R}+X_{F L}\right) \cos \theta_{F}+\left(X_{R R}+X_{R L}\right) \cos \theta_{R} \\
\quad-\left(Y_{F R}+Y_{F L}\right) \sin \theta_{F}-\left(Y_{R R}+Y_{R L}\right) \sin \theta_{R}, \\
m\left(\frac{d v}{d t}+u \gamma\right) \\
=\left(X_{F R}+X_{F L}\right) \sin \theta_{F}+\left(X_{R R}+X_{R L}\right) \sin \theta_{R} \\
\quad+\left(Y_{F R}+Y_{F L}\right) \cos \theta_{F}+\left(Y_{R R}+Y_{R L}\right) \cos \theta_{R},
\end{gathered}
$$




$$
\begin{array}{ll}
I \frac{d \gamma}{d t} & \\
= & l_{F}\left[\left(X_{F R}+X_{F L}\right) \sin \theta_{F}+\left(Y_{F R}+Y_{F L}\right) \cos \theta_{F}\right] \\
& +l_{R}\left[\left(X_{R R}+X_{R L}\right) \sin \theta_{R}+\left(Y_{R R}+Y_{R L}\right) \cos \theta_{R}\right] \\
& +\frac{d_{F}}{2}\left[\left(X_{F R}+X_{F L}\right) \cos \theta_{F}+\left(Y_{F R}+Y_{F L}\right) \sin \theta_{F}\right] \\
& +\frac{d_{R}}{2}\left[\left(X_{R R}+X_{R L}\right) \cos \theta_{R}+\left(Y_{R R}+Y_{R L}\right) \sin \theta_{R}\right] .
\end{array}
$$

7.2. Nonlinear Tire Characteristic. In the case of linear model, the forces acting on the vehicle is determined by the motions of the vehicle that are mainly side-slip angle and yaw rotational speed at the center of gravity. In our study, the acting forces are influenced by the wheels of the vehicle. Based on Fiala's theory, each wheel of the vehicle are modelled as a brush tire [14]. This theory assumes that with side-slip angle, the tire tread base deforms elastically in the lateral and longitudinal direction of the wheel. Due to this, the equations for friction and lateral forces are dependent on the value of wheels side-slip angle, slip ration, and friction coefficient. The deformation of the tire tread rubber is shown by the dimensionless variable $\xi_{i j}$ :

$$
\xi_{i j}=1-\frac{K_{\rho} \lambda_{i j}}{3 \mu_{i j} W_{z}\left(1-\rho_{i j}\right)},
$$

where

$$
\begin{aligned}
& \lambda_{i j}=\sqrt{\rho_{i j}^{2}+\left(\frac{K_{\beta}}{K_{\rho}}\right)^{2} \tan ^{2} \beta_{i j},} \\
& K_{\rho}=\frac{b l_{T}^{2}}{2} K_{x}, \\
& K_{\beta}=\frac{b l_{T}^{2}}{2} K_{y} .
\end{aligned}
$$

When $\xi_{i j}>0$, the contact surface comprises adhesive and slip region. Thus, longitudinal force and lateral force can be written as

$$
\begin{aligned}
X_{i j} & =-K_{\rho} \rho_{i j} \xi_{i j}{ }^{2}-6 \mu_{i j} W_{z} \frac{\rho_{i j}}{\lambda_{i j}}\left(\frac{1}{6}-\frac{1}{2} \xi_{i j}{ }^{2}+\frac{1}{3} \xi_{i j}{ }^{3}\right), \\
Y_{i j} & =-K_{\beta}\left(1+\rho_{i j}\right) \tan \beta_{i j} \xi_{i j}{ }^{2} \\
& -6 \mu_{i j} W_{z}\left(\frac{K_{\beta} \tan \beta_{i j}\left(1+\rho_{i j}\right)}{K_{\rho} \lambda_{i j}}\right) \\
& \cdot\left(\frac{1}{6}-\frac{1}{2} \xi_{i j}{ }^{2}+\frac{1}{3} \xi_{i j}{ }^{3}\right) .
\end{aligned}
$$

However, when $\xi_{i j} \leq 0$, the contact surface only holds the slip region. Therefore, the longitudinal force and lateral force can be written as

$$
\begin{aligned}
& X_{i j}=-\mu_{i j} W_{z} \frac{\rho_{i j}}{\lambda_{i j}} \\
& Y_{i j}=-\mu_{i j} W_{z}\left(\frac{K_{\beta} \tan \beta_{i j}\left(1+\rho_{i j}\right)}{K_{\rho} \lambda_{i j}}\right) .
\end{aligned}
$$

Here, the integral $i$ represents front and rear, while the integral $j$ represents right and left. The side-slip angle $\beta_{i j}$ which is mainly $\beta_{F R}, \beta_{F L}, \beta_{R R}, \beta_{R L}$ is given as follows:

$$
\begin{aligned}
& \beta_{F R}=\tan ^{-1}\left(\frac{v+l_{F} \gamma}{u+d_{F} \gamma / 2}\right)-\theta_{F}, \\
& \beta_{F L}=\tan ^{-1}\left(\frac{v+l_{F} \gamma}{u-d_{F} \gamma / 2}\right)-\theta_{F}, \\
& \beta_{R R}=\tan ^{-1}\left(\frac{v-l_{R} \gamma}{u+d_{R} \gamma / 2}\right)-\theta_{R}, \\
& \beta_{R L}=\tan ^{-1}\left(\frac{v-l_{R} \gamma}{u-d_{R} \gamma / 2}\right)-\theta_{R} .
\end{aligned}
$$

The slip ratio $\rho_{i j}$ can be defined as the traction between the road and the tire surface and is written as

$$
\rho_{i j}=\frac{r \omega_{i j}-u}{r \omega_{i j}} .
$$

Then, by using the slip ratio value, the coefficient of friction $\mu_{i j}$ can be approximated by the following equation:

$$
\mu_{i j}=-1.10 k \times\left(e^{35 p_{i j}}-e^{0.35 \rho_{i j}}\right),
$$

where

$$
\begin{aligned}
& k=1.0 \text { (dry asphalt), } \\
& k=0.2 \text { (icy road). }
\end{aligned}
$$

\section{State Observer Unit}

8.1. Linear Dynamic Equations. The ISCS consist of a state observer unit. This unit uses linear dynamic equations for the translational motion and yaw rotational motion such as

$$
\begin{aligned}
m v\left(\frac{d \beta^{*}}{d t}+\gamma^{*}\right) & =2 Y_{F}+2 Y_{R}, \\
I \frac{d \gamma^{*}}{d t} & =\left(2 l_{F} Y_{F}-2 l_{R} Y_{R}\right) \cos \beta^{*} .
\end{aligned}
$$

It is to be noted that there is no difference in the characteristics of the left and right tires due to linearity; hence, a 2-wheel model is derived from the equations. The sideslip angle $\beta^{*}$ is assumed to be small, which then lead to the assumption that the direction perpendicular to the traveling direction of the vehicle almost coincides with the lateral 
direction $y$. Therefore, no coupling exists between the yaw rotational speed and the vehicle's translational velocity.

The linear lateral force is defined by

$$
\begin{aligned}
& Y_{F}=-K_{F} \beta_{F}=-K_{F}\left(\beta^{*}+\frac{l_{F}}{v} \gamma^{*}-\theta_{F}\right), \\
& Y_{R}=-K_{R} \beta_{R}=-K_{R}\left(\beta^{*}-\frac{l_{R}}{v} \gamma^{*}-\theta_{R}\right) .
\end{aligned}
$$

Here, the cornering stiffness of the front and rear wheels is $K_{F}$ and $K_{R}$. These values determine the sensitivity of the ISCS [15]. In contrast, with the tire characteristics of the nonlinear model, the actual vehicle motion determines the linear model lateral forces, which are related to the sideslip angle, yaw rotational speed, and front and rear steering angles. The above equations can be substituted into the linear dynamic equations of motion (10) and the yaw rotational speed (11).

8.2. Linearized Differential Equation. The linearized differential equation represents the linear vehicle dynamic equation of motion for the state observer. It is arranged as a set of firstorder ordinary differential equations in the vector state form:

$$
\begin{aligned}
& \dot{x}=A x+B U_{R}+C U_{F}, \\
& y_{\beta}=\beta^{*}=\mathbb{C}_{1}^{T} x, \\
& y_{\gamma}=\gamma^{*}=\mathbb{C}_{2}^{T} x,
\end{aligned}
$$

where

$$
\begin{aligned}
& x=\left[\begin{array}{ll}
\beta^{*} & \gamma^{*}
\end{array}\right]^{T}, \\
& \mathbb{C}_{1}{ }^{T}=\left[\begin{array}{ll}
1 & 0
\end{array}\right]^{T} \text {, } \\
& U_{F}=\theta_{F} \text {, } \\
& \mathbb{C}_{2}{ }^{T}=\left[\begin{array}{ll}
0 & 1
\end{array}\right]^{T} \text {, } \\
& U_{R}=\theta_{R} \text {, } \\
& A=\left[\begin{array}{ll}
a & b \\
c & d
\end{array}\right] \\
& =\left[\begin{array}{cc}
-\frac{2\left(K_{F}+K_{R}\right)}{m v} & -1-\frac{2\left(K_{F} l_{F}-K_{R} l_{R}\right)}{m v^{2}} \\
-\frac{2\left(l_{F} K_{F}-l_{R} K_{R}\right)}{I} & \frac{2\left(l_{F}{ }^{2} K_{F}+l_{R}{ }^{2} K_{R}\right)}{I v}
\end{array}\right] \text {, } \\
& B=\left[\begin{array}{l}
e \\
f
\end{array}\right]=\left[\begin{array}{c}
\frac{2 K_{R}}{m v} \\
-\frac{2 l_{R} K_{R}}{I}
\end{array}\right] \text {, } \\
& C=\left[\begin{array}{l}
k \\
l
\end{array}\right]=\left[\begin{array}{c}
\frac{2 K_{F}}{m v} \\
\frac{2 l_{F} K_{F}}{I}
\end{array}\right] \text {. }
\end{aligned}
$$

TABLE 1: Weighting matrices characteristic.

\begin{tabular}{lcccc}
\hline $\begin{array}{l}\text { Large } \\
\text { value }\end{array}$ & Constant & $|x|$ & $|\dot{x}|$ & $|U|$ \\
\hline$q_{11}$ & $q_{22}, w$ & Reduce & Increase & Increase \\
$q_{22}$ & $q_{11}, w$ & Increase & Reduce & Increase \\
$w$ & $q_{11}, q_{22}$ & Increase & Increase & Reduce \\
\hline
\end{tabular}

The state vector $x$ represents the side-slip angle and yaw rotational speed of the vehicle. The steering angles of the front and rear wheels are the inputs of this system. The value for the front wheel angle $\theta_{F}$ is a driver-selected input whereas the rear wheel angle $\theta_{R}$ is initially 0 degrees. During high-speed cornering, the rear wheel steering is used as the control input by introducing a feedback gain $-g^{T}$ :

$$
U_{R}=-g^{T} x+\theta_{R}
$$

8.3. Optimal Control. In order to find the optimal control for the linearized differential equation, the following evaluation function $J$ is employed:

$$
\begin{aligned}
J & =\int_{0}^{\infty}\left(q_{11} x^{2}+q_{22} \dot{x}^{2}+w \theta_{r}^{2}\right) d t \\
& =\int_{0}^{\infty}\left(x^{T} Q x+w \theta_{r}^{2}\right) d t,
\end{aligned}
$$

where

$$
Q=\left[\begin{array}{cc}
q_{11} & 0 \\
0 & q_{22}
\end{array}\right]
$$

in which $q_{11}, q_{22}$, and $w$ are appropriately chosen constant weighting matrices for the state vector $x$, the first-order differential of state vector $\dot{x}$, and the control input $U_{R}$, respectively. Table 1 shows the characteristics of the weighting matrices value that control the state and control value. The control input has its own high gain $H$ and thus the weighting matric $w=1$. The weighting matrices of $q_{11}$ and $q_{22}$ were used to stabilized the state vector, which is yaw rotational speed and side-slip angle to prevent it from reaching infinite value by the oversteering characteristic of the vehicle. The control input weighting matric $w=1$.

The optimal solution for $J$ can be designed if $Q$ is a positive definite matrix and the state observer control input $U_{R}$ is given by

$$
U_{R}=-\frac{1}{w} B^{T} P x+\theta_{R}
$$

where

$$
-g^{T}=-\frac{1}{w} B^{T} P
$$

With $P=P^{T} \geq 0$ being the unique positive-semidefinite solution of the algebraic Riccati equation,

$$
\begin{aligned}
& A^{T} P+P A-w^{-1} P B B^{T} P=-Q, \\
& {\left[\begin{array}{ll}
a & b \\
c & d
\end{array}\right]^{T} P+P\left[\begin{array}{ll}
a & b \\
c & d
\end{array}\right]-w^{-1} P\left[\begin{array}{l}
e \\
f
\end{array}\right]\left[\begin{array}{ll}
e & f
\end{array}\right]^{T} P} \\
& \quad=-\left[\begin{array}{ll}
q_{1} & 0 \\
0 & q_{2}
\end{array}\right] .
\end{aligned}
$$


The unique positive-semidefinite $P$ can also be expressed as

$$
P=\left[\begin{array}{ll}
\varepsilon & \phi \\
\psi & \epsilon
\end{array}\right] .
$$

Thus, (20) can be expanded as

$$
\begin{aligned}
a \varepsilon & +c \psi+a \varepsilon+c \phi \\
& -\frac{1}{w}\left(\left[e^{2} \varepsilon+e f \phi\right] \varepsilon+\left[e f \varepsilon+f^{2} \phi\right] \psi\right)=-q_{11}, \\
a \phi & +c \epsilon+b \varepsilon+d \phi \\
& -\frac{1}{w}\left(\left[e^{2} \varepsilon+e f \phi\right] \phi+\left[e f \varepsilon+f^{2} \phi\right] \epsilon\right)=0, \\
b \varepsilon & +d \psi+a \psi+c \epsilon \\
\quad & -\frac{1}{w}\left(\left[e^{2} \psi+e f \epsilon\right] \varepsilon+\left[e f \psi+f^{2} \epsilon\right] \psi\right)=0, \\
b \phi & +d \epsilon+b \psi+d \epsilon \\
& -\frac{1}{w}\left(\left[e^{2} \psi+e f \epsilon\right] \phi+\left[e f \psi+f^{2} \epsilon\right] \epsilon\right)=-q_{22} .
\end{aligned}
$$

Finally, the output of the vehicle model and the state observer was compared to form a close loop system. In general, there is no other method to directly measure the output of side-slip angle. However, yaw rotational speed of a vehicle can be calculated with gyro sensors. In this system, the estimated yaw rotational speed output of state observer unit and the measured yaw rotational speed output of the vehicle model are compared and multiplied by high gain $H$. Then, this gain is fed back into the linear model. The linearized differential equation of (13) becomes

$$
\begin{aligned}
& \dot{\dot{x}}=A x+B U_{R}+C U_{f}+H \widetilde{r}, \\
& \tilde{r}=\gamma-\gamma^{*}=\mathbb{C}_{2}^{T} \widehat{x}-\mathbb{C}_{2}{ }^{T} x=\mathbb{C}_{2}{ }^{T}(\widehat{x}-x) .
\end{aligned}
$$

The renewed state observer control input can also be redefined as follows:

$$
U_{R}=-\frac{1}{r} B^{T} P(\widehat{x}-x)+\theta_{R} .
$$

With this equation, the FFC for the 4WDIS in-wheel small $\mathrm{EV}$ can be changed to a FBC that gives greater precision.

\section{Simulation Procedures}

9.1. Determine the Vehicle Model Steering Characteristic. In the previous section, we mentioned that the vehicle model used the Toyota COMS specification and modified it to represent an OS in-wheel small electric vehicle in the simulation. A simulation of a steady-state cornering at various velocities with regard to a constant front steer angle was done to determine the stability limit velocity of the vehicle model before 4 WDIS could be implemented. The constant front steer angle is fix at $10^{\circ}$ at $t=10 \mathrm{~s}$. When the yaw rotational speed and the side-slip angle give an infinite value, the velocity before that moment is acknowledged as the stability limit velocity.

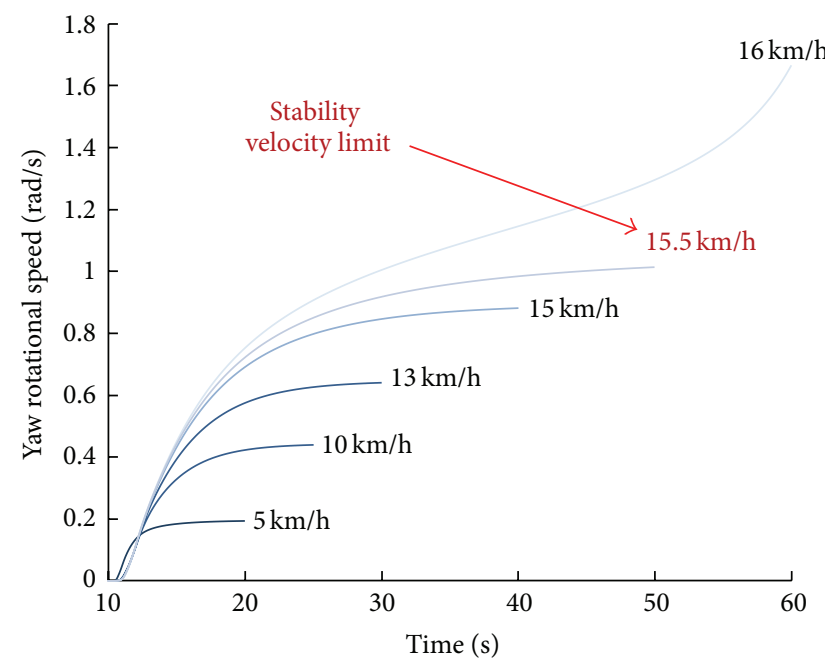

FIGURE 7: Steady-state yaw rotational speed to time change with 2WS.

9.2. Implementation of 4 WDIS with ISCS. A 4 WDIS for OS in-wheel small EV was constructed as shown is previous simulation block diagram. In order to investigate the performance of 4WDIS in depth, we have divided the simulation as follows:

(1) Steady-state cornering below stability limit velocity.

(2) Steady-state cornering over stability limit velocity.

Similar to previous simulation condition, a steady-state cornering of a 4 WDIS in-wheel small EV at below stability limit velocity and over stability limit velocity, with regard to a constant front steer angle, was carried out. The constant front steer angle is fix at $10^{\circ}$ at $t=10 \mathrm{~s}$. The results of the steadystate yaw rotational speed and steady-state side-slip angle for 4WDIS are compared to the previous simulation.

\section{Results and Discussion}

10.1. Stability Velocity Limit of the OS Vehicle Model during 2 WS Cornering. A simulation of steady-state cornering was carried out to determine the stability limit velocity of the OS vehicle model. Figures 7 and 8 show the results of the steadystate yaw rotational speed and steady-state side-slip angle with regard to constant front wheel steer angle, respectively. Based on both of these graphs, in regard to constant front steer angle of $10^{\circ}$, the stability limit velocity $V_{c}$ is $15.5 \mathrm{~km} / \mathrm{h}$. At this velocity, the vehicle is stable and can reach the maximum steady-state yaw rotational speed of $1.01 \mathrm{rad} / \mathrm{s}$ with a maximum absolute magnitude of the steady-state side-slip angle of $0.301 \mathrm{rad}$. When the velocity is above $15.5 \mathrm{~km} / \mathrm{h}$, the yaw rotational speed and the side-slip angle will give an infinite value. However, the vehicle can still be driven but it is very unstable. This shows that we have successfully modelled a highly OS in-wheel small EV.

Nonetheless, we believe that even in the stable region which is below the stability limit velocity, the OS small EV can be improved. In Figure 7, when the constant velocity 


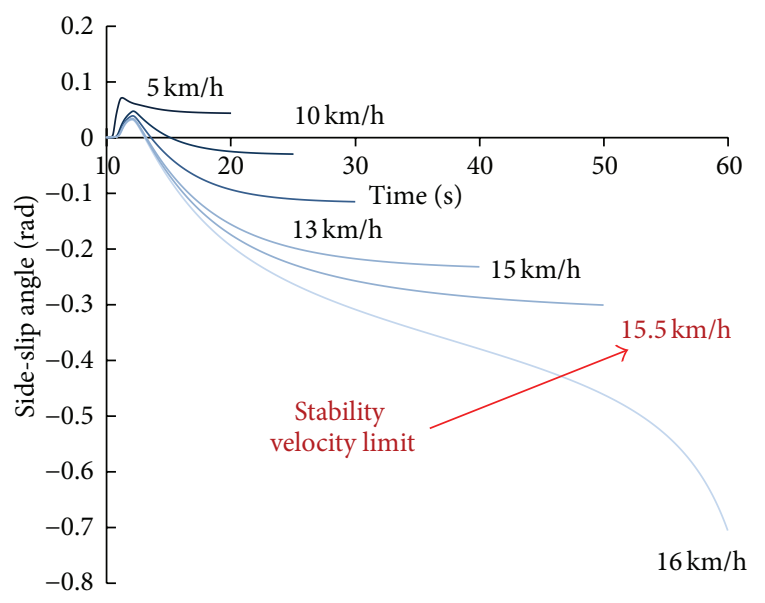

FIGURE 8: Steady-state side-slip angle to time change with 2WS.

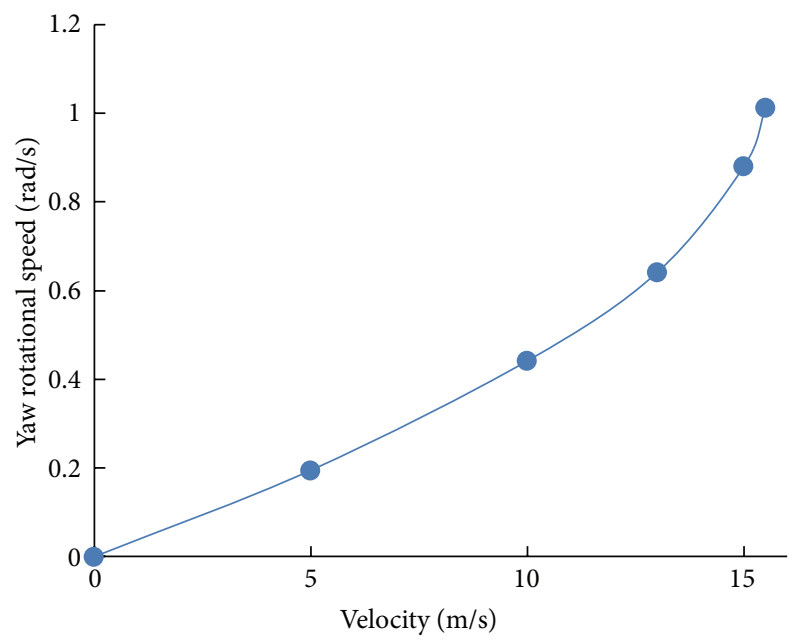

FIGURE 9: Steady-state yaw rotational speed to velocity with 2WS.

increased, the vehicle model took longer time to generate a constant yaw rotational speed. This is due to the increase in side-slip angle to the constant velocity. The relation between steady-state yaw rotational speed and velocity is plotted in Figure 9, while the relation between steady-state side-slip angle and velocity is plotted in Figure 10.

10.2. Steady-State Cornering below Stability Limit Velocity with 4 WDIS. Theoretically, OS vehicles can already produce high yaw rotational speed and stability when cornering below the stability limit velocity. Logically, any control system is unnecessary in the stable region. We can assume that the cornering performance in the stable region of the OS in-wheel small EV will be suppressed by implementing the 4WDIS.

In this section, the control effect of 4WDIS implementation onto an OS in-wheel small EV during steady-state cornering below the stability limit velocity in regard to constant front steer angle $10^{\circ}$ is examined. Figures 11 and 12 show the results of the steady-state yaw rotational speed and steady-state side-slip angle for the vehicle model with 4 WDIS, respectively. The average time for the yaw rotational

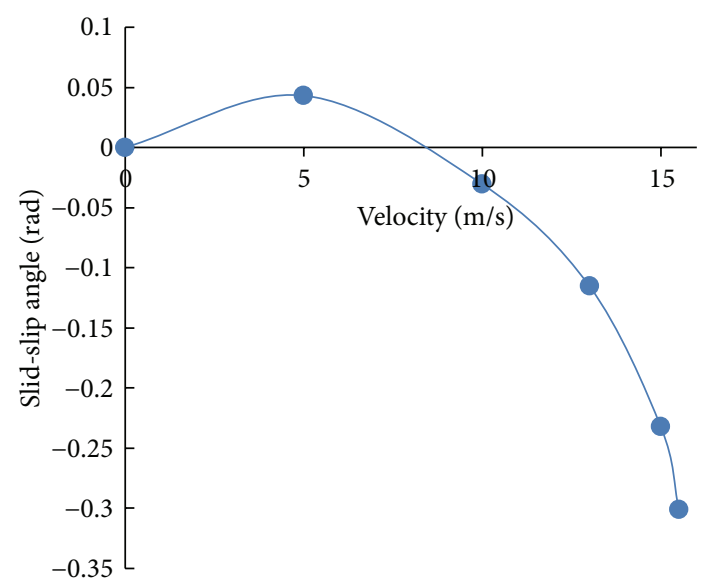

FIGURE 10: Steady-state side-slip angle to velocity with 2WS.

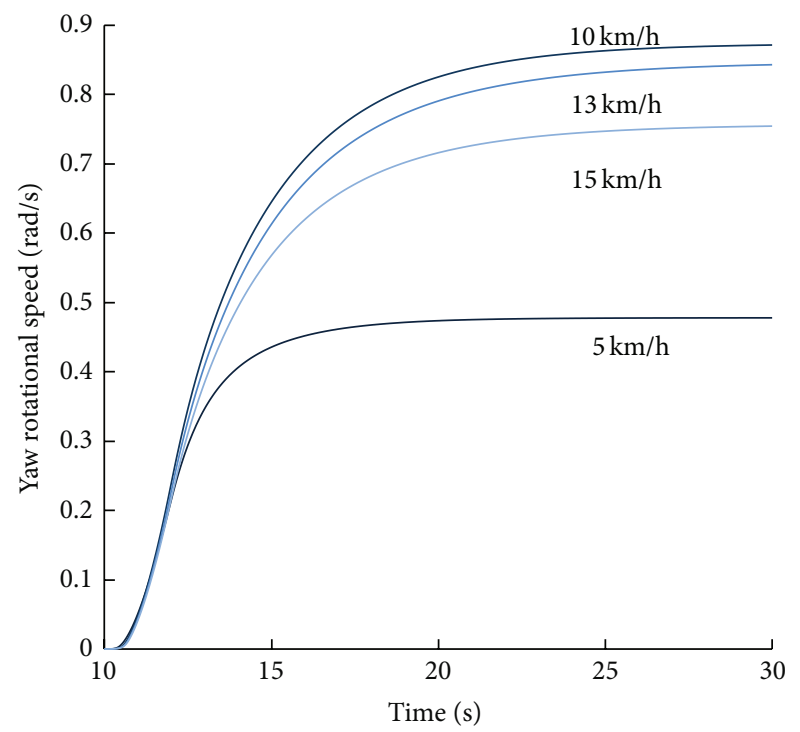

FIGURE 11: Steady-state yaw rotational speed to time change for the vehicle model with 4WDIS.

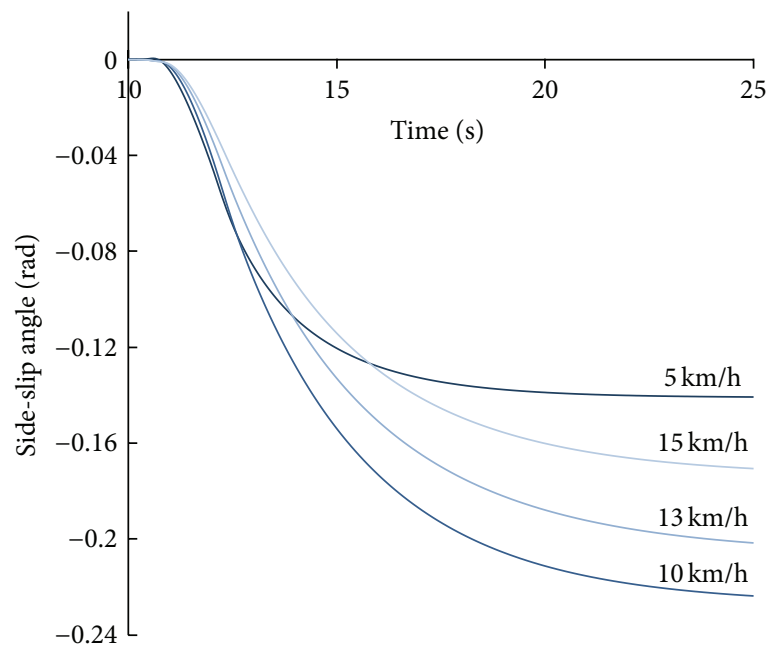

FIGURE 12: Steady-state side-slip angle to velocity for the vehicle model with 4WDIS. 


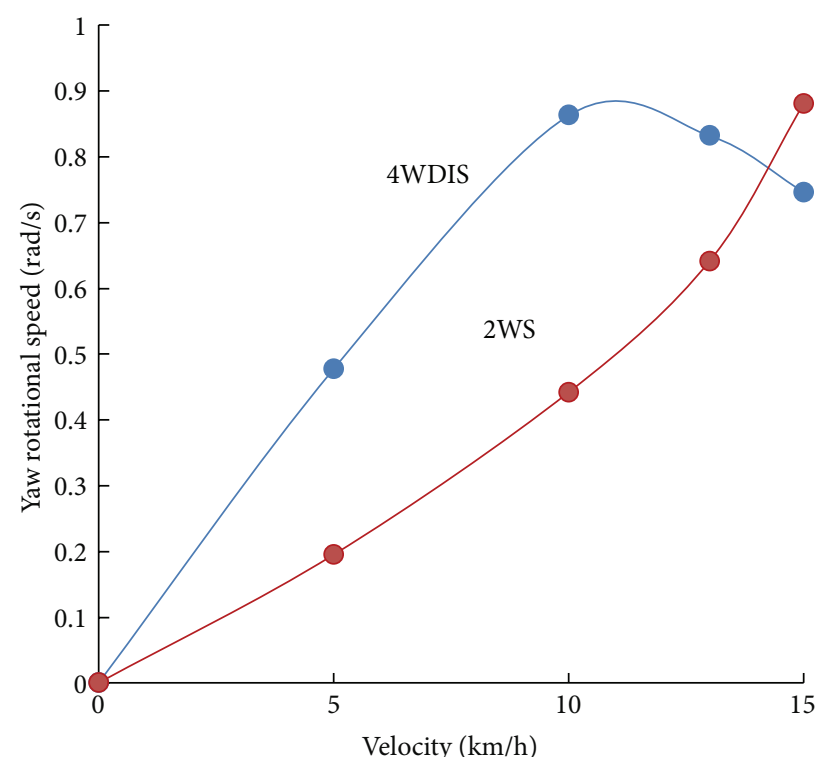

FIGURE 13: Comparison of steady-state yaw rotational speed to velocity for $2 \mathrm{WS}$ and $4 \mathrm{WDIS}$.

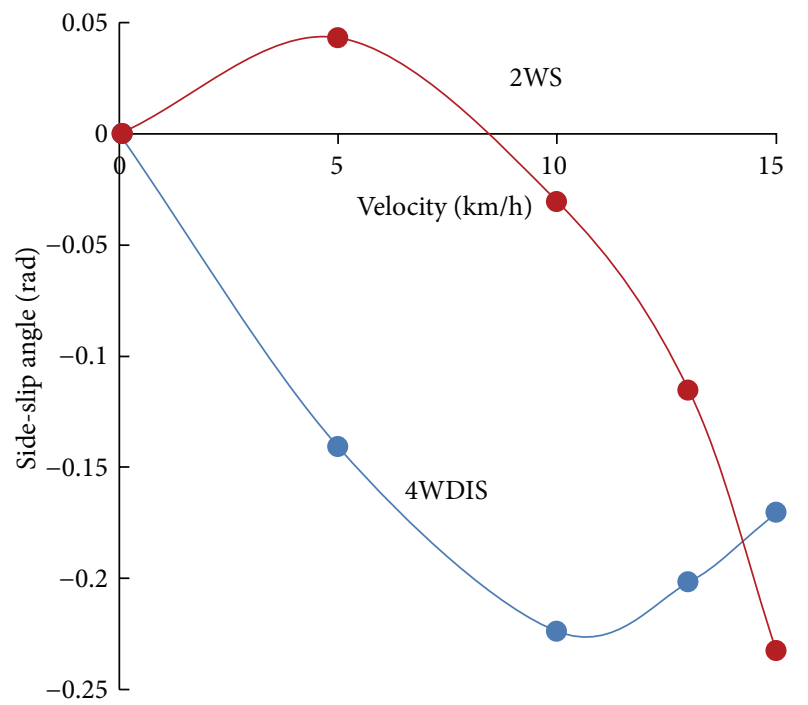

FIGURE 14: Comparison of steady-state side-slip angle to velocity for 2WS and 4WDIS.

speed to reach a constant value was also reduced to $25 \mathrm{~s}$ in comparison to the $2 \mathrm{WS}$ vehicle model. Even with high yaw rotational speed, the steady-state side-slip angle also remains constant. The steady-state side-slip angle was in negative values which indicate that the vehicle was facing the inner circle of the cornering direction as the cornering initiated.

For better comparison, the relation between steady-state yaw rotational speed to velocity and steady-state side-slip angle to velocity for vehicle model with 4 WDIS and 2WS was plotted in Figures 13 and 14. The steady-state yaw rotational speed of the vehicle model with 4WDIS was approximately twofold the value of vehicle model with normal 2WS at velocity range of 0 to $10 \mathrm{~km} / \mathrm{h}$. When the velocity of the vehicle

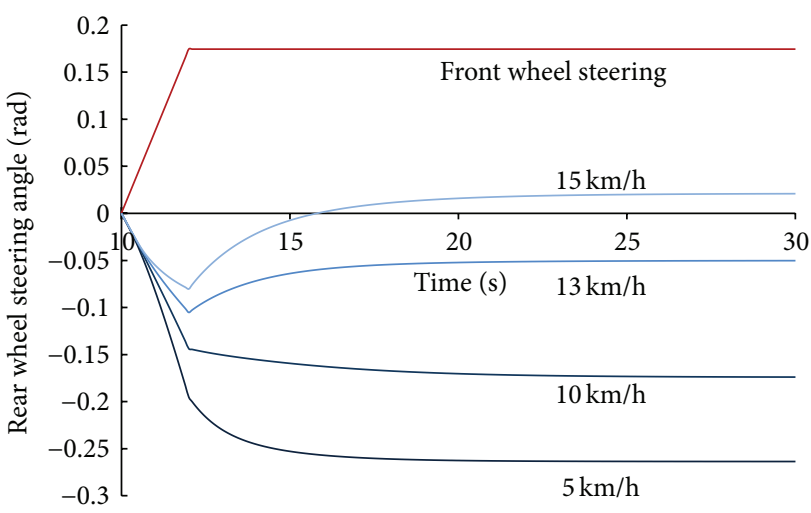

FIGURE 15: The front and rear wheel steering angle during the steadystate cornering at below stability limit velocity for the vehicle model with 4WDIS.

increased from $10 \mathrm{~km} / \mathrm{h}$ to $15 \mathrm{~km} / \mathrm{h}$, the steady-state yaw rotational speed gradually decreased. A similar pattern occurred for the side-slip angle if we observe in absolute magnitude.

In order to comprehend these matters, we have to examine the output of the ISCS. Figure 15 shows the rear wheel steering angle during the steady-state cornering of the 4WDIS vehicle model. As soon as the front wheel steer input initiated at $t=10 \mathrm{~s}$, the rear wheel steer initiated at the opposite direction as the front wheel. The rear wheel steering angle maintained an opposite angle as the front wheel which increased the yaw rotational speed. As the velocity approach near the stability limit, the opposite angle of the rear wheel decreased.

10.3. Steady-State Cornering over Stability Limit Velocity with $4 W D I S$. The stability limit velocity of the modelled OS in-wheel small EV has been determined in the previous simulation. The previous simulation also proves that the implementation of 4WDIS is able to improve the maneuverability of the OS small electric vehicle even at stable region. The more anticipated matter is the control of 4WDIS for the OS small electric vehicle during cornering above the stability velocity limit. The previous steady-state cornering simulation condition is repeated again for this vehicle model.

In this section, the control effect of the 4WDIS on an OS in-wheel small EV during steady-state cornering over the stability limit velocity in regard to constant front steer angle $10^{\circ}$ is investigated. Figure 16 shows the result of the steady-state yaw rotational speed for the vehicle model with 4WDIS. From this figure, the yaw rotational speed decreased as the velocity of the vehicle model increased. However, if we observe closely, the time to produce a constant steadystate yaw rotational speed is instantaneous and maintains the average time of $25 \mathrm{~s}$, especially at velocity range of 30 to $50 \mathrm{~km} / \mathrm{h}$. The results of steady-state side-slip angle for this simulation in Figure 17 show a reverse pattern from the yaw rotational speed. The side-slip angle increased as the velocity of the vehicle model increased. Based on these results, we can assumed that the 4WDIS control input suppressed the output more as the velocity of the vehicle model increased. 


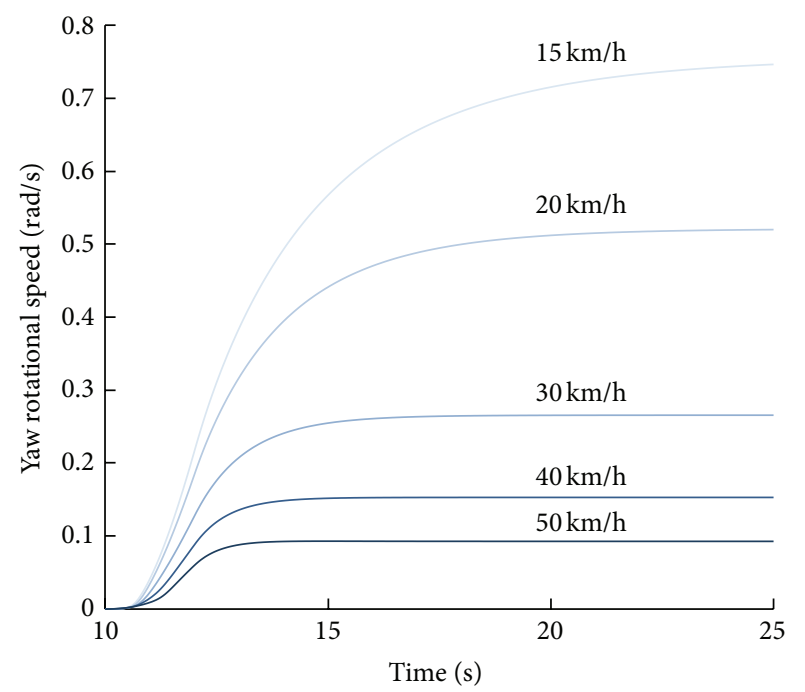

FIGURE 16: Steady-state yaw rotational speed to time change for the vehicle model with 4 WDIS.

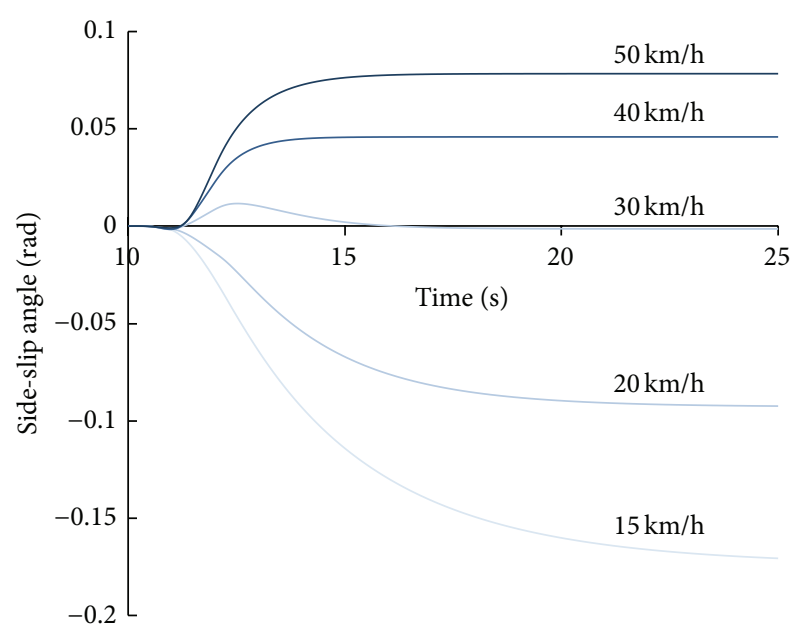

FIGURE 17: Steady-state side-slip angle to time change for the vehicle model with 4WDIS.

In order to understand the control method of the 4WDIS by the ISCS, we have to examine the output of the system. Figure 18 shows the front and rear wheel steering angle during the steady-state cornering simulation of the 4WDIS vehicle model. In the first few second after the front wheel steering angle initiated, the ISCS produced a rear wheel steering angle in minus value that gave the instantaneous yaw rotational speed mentioned before. The minus angle of the rear wheel was smaller as the steady-state velocity increased because the excess yaw rotational speed produced by OS vehicle model also increased. As time progressed, the ISCS gradually increased the rear wheel steering angle to counter the excess yaw rotational speed and sustain a constant value. The angle of the rear wheel is higher for velocity range over $15 \mathrm{~km} / \mathrm{h}$ which proves that our early assumption was correct.

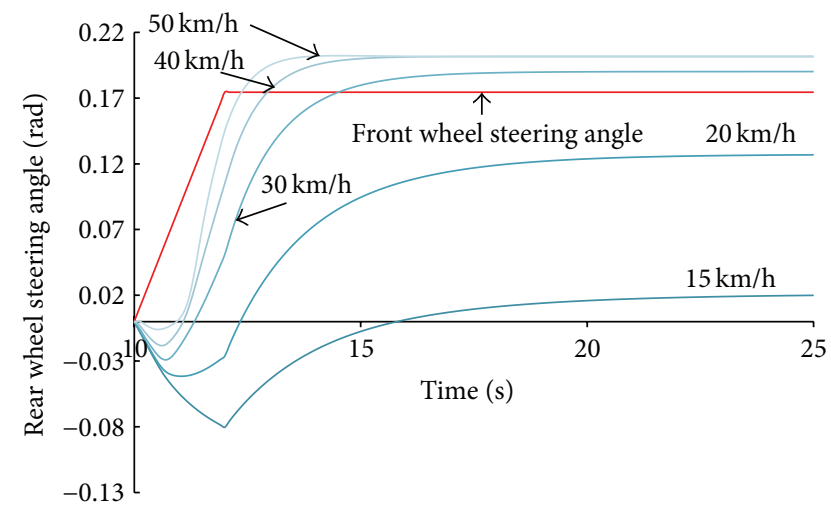

FIGURE 18: The front and rear wheel steering angle during the steadystate cornering at over stability limit velocity for the vehicle model with 4WDIS.

10.4. Overall Comparison of Steady-State Cornering with $2 W S$ and 4 WDIS. Figures 19(a) and 19(b) show the characteristics of the steady-state yaw rotational speed and side-slip angle for an in-wheel small EV integrated with our modelled 4WDIS control in comparison to the conventional 2WS vehicle, respectively. This figure is a combination of the simulation results of the 4WDIS control simulation.

Based on these results, an OS in-wheel small EV can achieve a stable steady-state cornering even when the velocity is over the stability limit velocity with the implementation of 4WDIS. Simply put, the 4WDIS eliminate the stability velocity that was present in the OS small electric vehicle with $2 \mathrm{WS}$. The 4WDIS control input suppressed the yaw rotational speed and side-slip angle during high speed by rotating the rear wheels at the same direction as the front wheels. Additionally, it can also improve the maneuverability of the vehicle at low speed by rotating the rear wheels at the opposite direction of the front wheels.

\section{Conclusion}

There are various methods to design an OS vehicle. In this paper, we chose the transfer of weight distribution method to model an OS in-wheel small EV. There are also a few ways to recognize an OS vehicle and one of them is that the vehicle will have a stability limit velocity. When a steady-state cornering simulation was carried out below the stability limit velocity $V_{c}$, the OS in-wheel small EV can achieve steadystate cornering with a higher yaw rotational speed with regard to constant low steering input. However, when the vehicle velocity $V \geq V_{c}$, the yaw rotational speed and the side-slip angle produced an infinite value in regard to the same constant steer angle.

The main objective of implementing 4WDIS onto an OS in-wheel small EV is to ensure stability during cornering at any velocity. The $4 \mathrm{WDIS}$ utilized the opposite at low speed to gain yaw motion and parallel steering to reduce the yaw motion of the vehicle.

In this paper, the Intelligence Steering Control System (ISCS) evaluated the dynamic of the in-wheel small EV and dry road surface. In the future, other parameters such as 


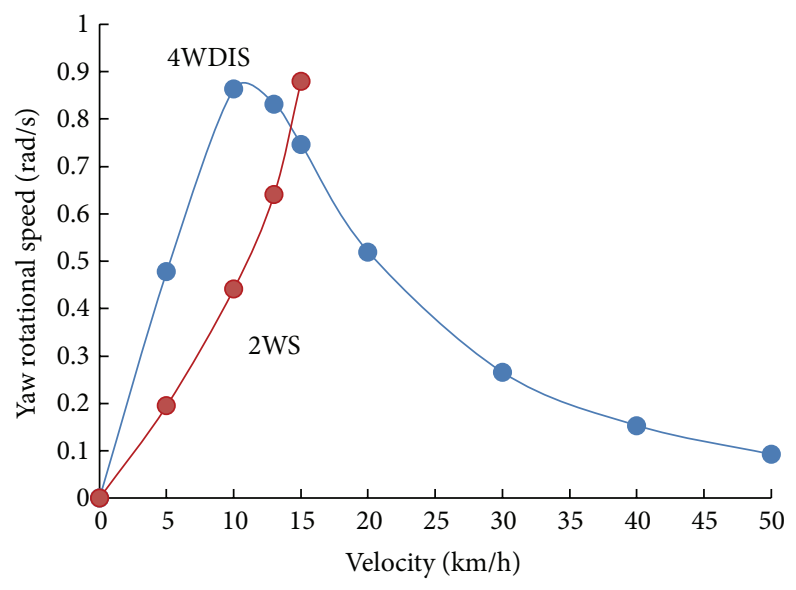

(a)

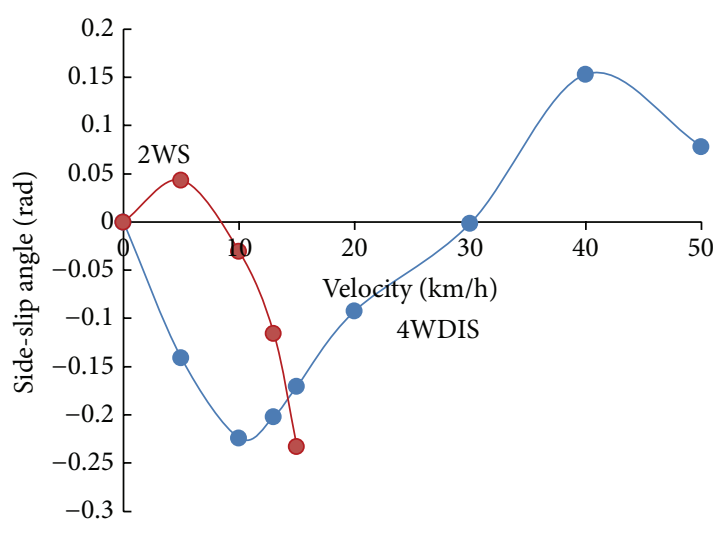

(b)

FIGURE 19: (a) Steady-state yaw rotational speed to velocity for the vehicle model with 4WDIS. (b) Steady-state side-slip angle to velocity for the vehicle model with 4 WDIS.

lateral disturbance or icy road surface which has effect on the driving or the vehicle will be taken into consideration in the simulation analysis.

\section{Nomenclature}

Constant Parameters Symbols, Meaning, Value, and Unit

$b$ : Width of wheel interact surface, $0.1, \mathrm{~m}$

$d_{F}$ : Front tread, $0.840, \mathrm{~m}$

$d_{R}$ : Rear tread, $0.815, \mathrm{~m}$

I: Yaw inertia moment at gravity point of vehicle, $1470, \mathrm{kgm}^{2}$

$K_{x}$ : Longitudinal tread rubber stiffness, $3.33 \times$ $10^{6}, \mathrm{~N} / \mathrm{m}^{3}$

$K_{y}$ : Lateral tread rubber stiffness, $3.33 \times 10^{6}$, $\mathrm{N} / \mathrm{m}^{3}$

$K_{F}$ : Front wheel cornering stiffness, 2000.00, $\mathrm{N} / \mathrm{rad}$

$K_{R}$ : Rear wheel cornering stiffness, 2612.62 , $\mathrm{N} / \mathrm{rad}$

$l_{T}$ : Length of wheel interact surface, $0.15, \mathrm{~m}$

$l_{F}$ : Length from front axle wheel to gravity point of vehicle, $0.725, \mathrm{~m}$

$l_{R}$ : Length from rear wheel axle to gravity point of vehicle, $0.555, \mathrm{~m}$

$l$ : Length from front axle to rear axle of the wheel, $1.28, \mathrm{~m}$

$m$ : Mass of the vehicle, $421.61, \mathrm{~kg}$

$r$ : Radius of the wheel, $0.23, \mathrm{~m}$.

Variables Symbols, Meaning, Value, and Unit

$\begin{array}{ll}u: & \text { Longitudinal velocity, } \mathrm{m} / \mathrm{s} \\ v: & \text { Lateral velocity, } \mathrm{m} / \mathrm{s} \\ V: & \text { Velocity at center gravity, } \mathrm{m} / \mathrm{s} \\ V_{c}: & \text { Stability limit velocity, } \mathrm{m} / \mathrm{s} \\ X_{F R}, X_{F L}, X_{R R}, X_{R L}: & \text { Friction force for each tire, } \mathrm{N}\end{array}$

$Y_{F R}, Y_{F L}, Y_{R R}, Y_{R L}:$ Lateral force for each tire, $\mathrm{N}$

$W_{z}$ : $\quad$ Wheels load subject to load transfer, $\mathrm{N}$

$\beta: \quad$ Side-slip angle of vehicle, rad

$\beta^{*}$ : $\quad$ Side-slip angle of state observer, rad

$\beta_{F R}, \beta_{F L}, \beta_{R R}, \beta_{R L}$ : Side-slip angle of each wheel, rad

$\mu$ : $\quad$ Friction coefficient

$\theta_{F}, \theta_{R}: \quad$ Front and rear wheels steer angle, rad

$\gamma: \quad$ Yaw rotational speed of vehicle body,

$\gamma^{*}: \quad \quad$ Yaw rotational speed of state observer,

$\rho: \quad$ rad $/ \mathrm{s}$

$\omega: \quad$ Wheel rotational speed, $\mathrm{rad} / \mathrm{s}$.

\section{Competing Interests}

The authors declare that they have no competing interests.

\section{References}

[1] Ministry of Land Infrastructure and Transport Department of Municipal Affairs and Automobile Station, "Towards the realization of a new social life through the development and utilization of new (mobility) guidelines for the introduction of ultrasmall mobility," 2010, http://www.mlit.go.jp/common/001129801 .pdf.

[2] Y. Hori, "Future vehicle driven by electricity and controlresearch on four wheel motored 'UOT Electric March II"' in Proceedings of the 7th International Workshop on Advanced Motion Control (AMC '02), pp. 1-14, Maribor, Slovenia, July 2002.

[3] M. Izhar, H. Ogino, and Y. Oshinoya, "Research on motion control of 4 wheels steering vehicles-effect of regeneraive brake on vehicles motion," Proceeding of The School of Engineering, Tokai University, vol. 53, no. 2, pp. 99-103, 2013.

[4] M. Izhar, H. Ogino, and Y. Oshinoya, "Research on 4 wheels independent steering for small electric vehicle (effect of stability clearance speed)," in Proceedings of the Japan Society of Mechanical Engineers Annual Yearly Conference, No. 14, 2014. 
[5] H. Ogino, M. Izhar, and Y. Oshinoya, "Stability analysis of small electric vehicle (effect of hysteresis of friction brake force for regenerative braking force)," Proceeding of The School of Engineering, Tokai University, vol. 52, pp. 55-64, 2012.

[6] R. Bundorf, "The influence of vehicle design parameters on characteristic speed and understeer," SAE Technical Paper 670078, 1967.

[7] M. Abe, "Vehicle handling dynamics: theory and application," in Vehicle Handling Dynamics: Theory and Application, p. 61, Butterworth-Heinemann, 2nd edition, 2009.

[8] T. Murphy and B. Corbett, “Quadrasteer off course," Ward's Auto World, 2005.

[9] E. Ona, Y. Hattori, H. Aizawa, H. Kato, S. Tagawa, and S. Niwa, "Clarification and achievement of theoratical limitation in vehicle dynamics integrated management," Journal of Environment and Engineering, vol. 4, no. 1, pp. 89-100, 2009.

[10] F. Du, J.-S. Li, L. Li, and D.-H. Si, "Robust control study for fourwheel active steering vehicle," in Proceedings of the International Conference on Electrical and Control Engineering (ICECE '10), pp. 1830-1833, June 2010.

[11] N. Hamzah, M. K. Aripin, Y. M. Sam, H. Selamat, and M. F. Ismail, "Yaw stability improvement for four-wheel active steering vehicle using sliding mode control," in Proceedings of the IEEE 8th International Colloquium on Signal Processing and Its Applications (CSPA '12), pp. 127-132, IEEE, Melaka, Malaysia, March 2012.

[12] J. Tian, N. Chen, J. Yang, and L. Wang, "Fractional order sliding model control of active four-wheel steering vehicles," in Proceedings of the International Conference on Fractional Differentiation and Its Applications (ICFDA '14), pp. 1-5, IEEE, Catania, Italy, June 2014.

[13] S.-H. Yu and J. J. Moskwa, "A global approach to vehicle control: coordination of four wheel steering and wheel torques," Journal of Dynamic Systems, Measurement, and Control, vol. 116, no. 4, pp. 659-667, 1994.

[14] M. Abe, Vehicle Handling Dynamics: Theory and Application, Butterworth-Heinemann, Boston, Mass, USA, 2nd edition, 2009.

[15] M. Izhar, H. Ogino, and Y. Oshinoya, "Research on 4 wheel drive and independent steering for small electric vehicle: active stability control during high speed cornering," Proceeding of the School of Engineering, Tokai University, vol. 40, pp. 63-70, 2015. 


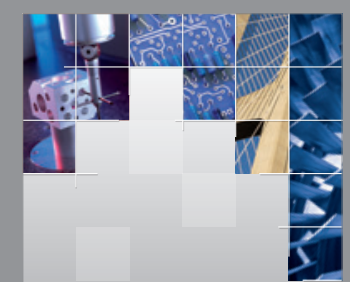

\section{Enfincering}
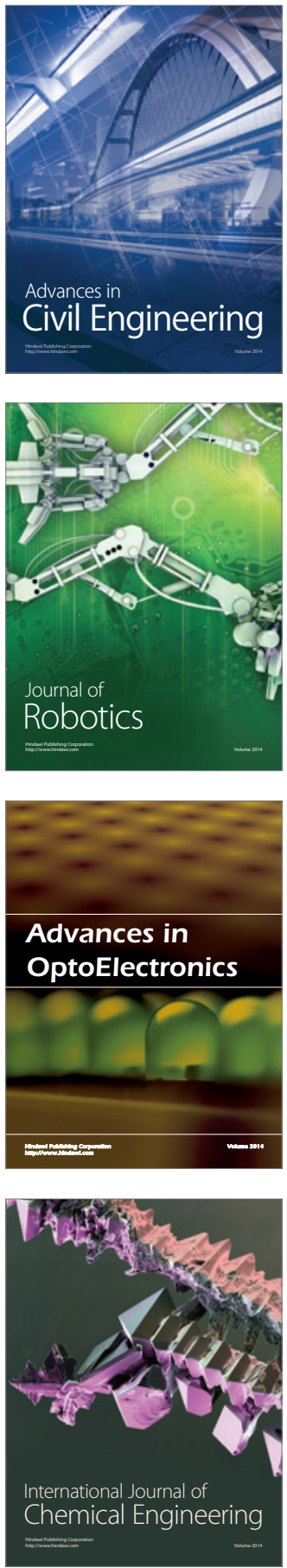

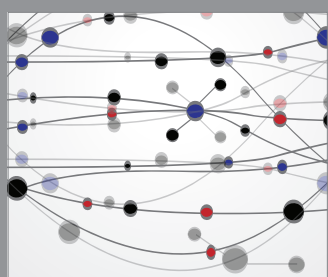

The Scientific World Journal

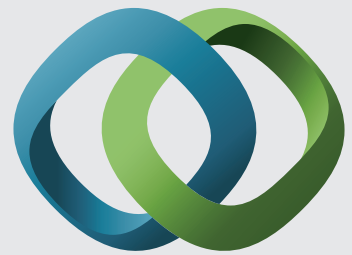

\section{Hindawi}

Submit your manuscripts at

http://www.hindawi.com
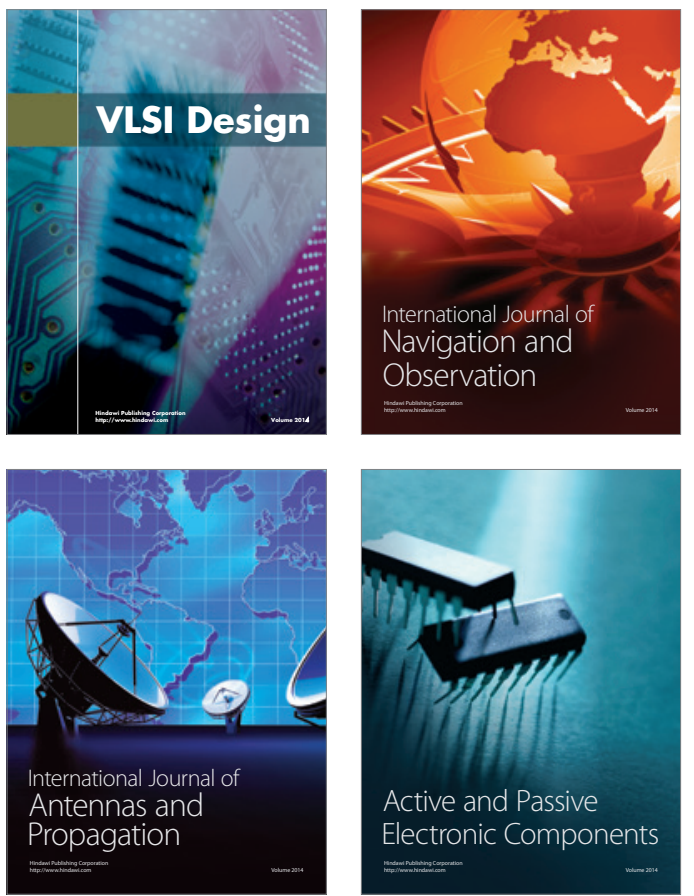
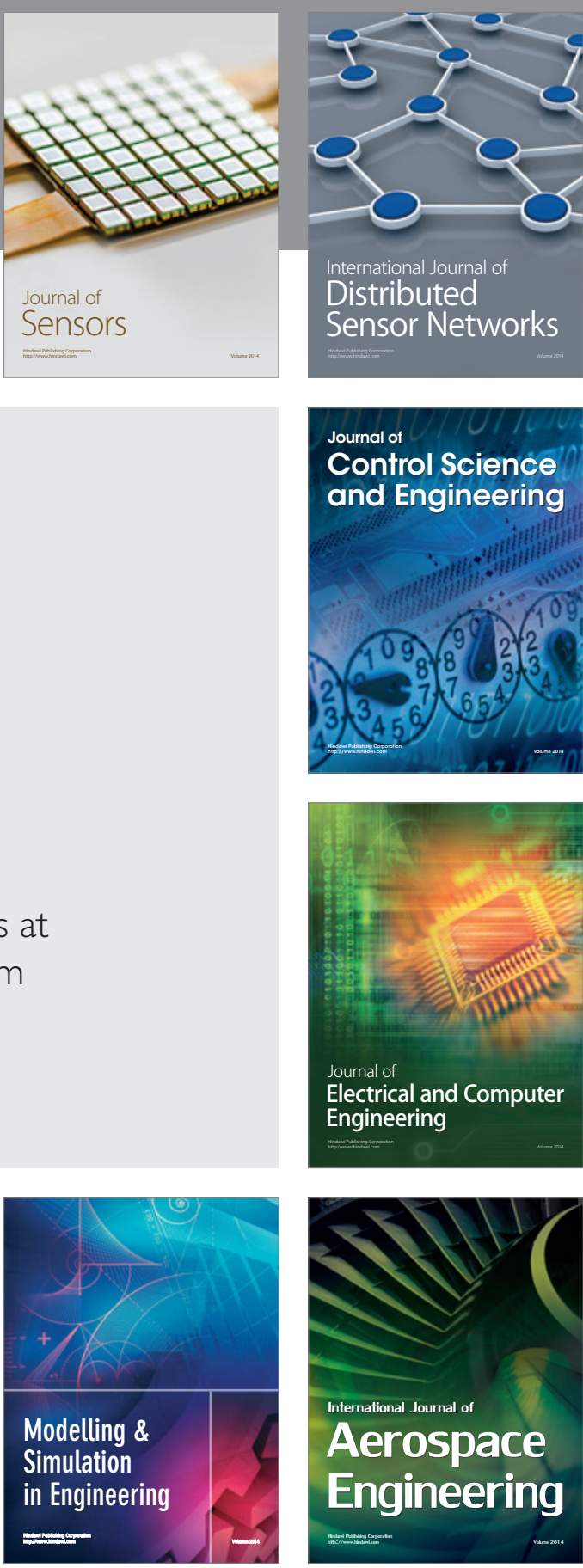

International Journal of

Distributed

Sensor Networks

Journal of

Control Science

and Engineering
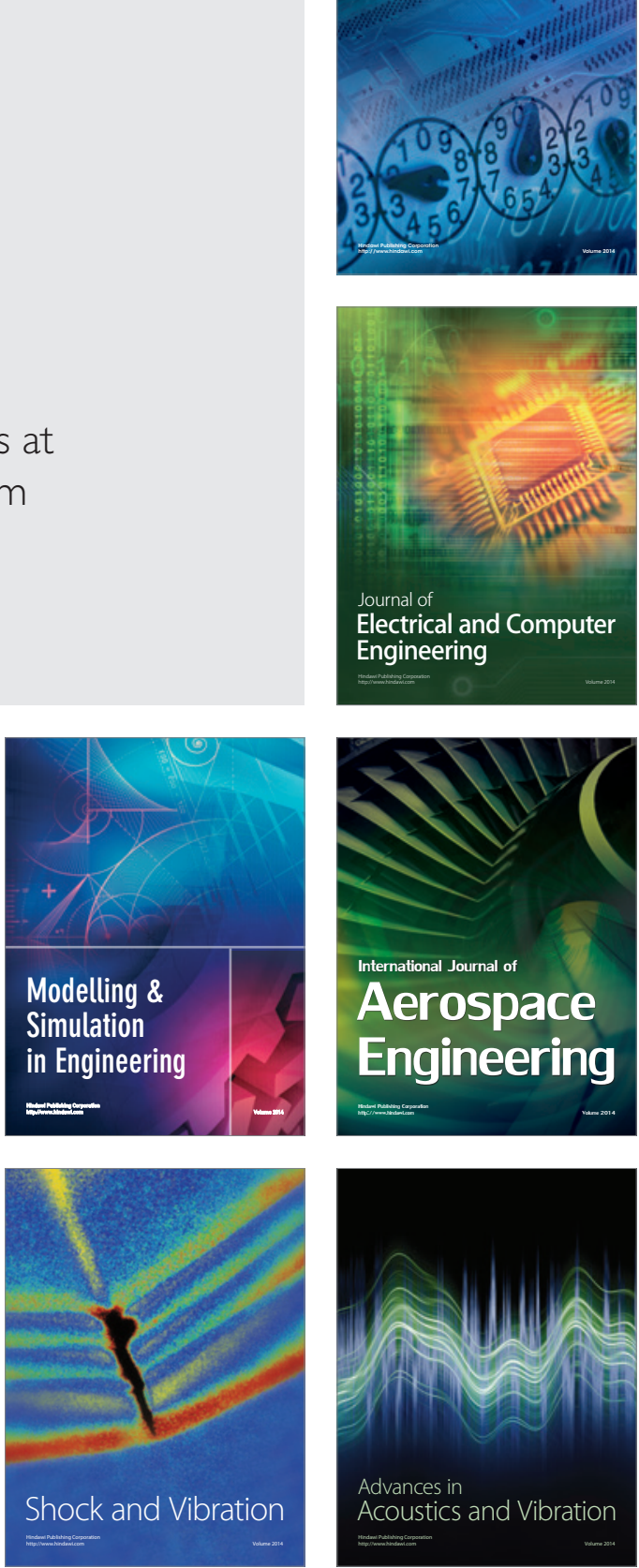